Encontros Bibli: revista eletrônica de biblioteconomia e ciência da informação, v. 17, $n$. 34, p.31-41, maio./ago., 2012. ISSN 1518-2924. DOI: 10.5007/1518-2924.2012v17n34p31

\title{
CONSTITUIÇÃO DA MICOBIOTA AÉREA DE BIBLIOTECAS PÚBLICAS NO MUNICÍPIO DE FORTALEZA, ESTADO DO CEARÁ, BRASIL.
}

\author{
Lydia Dayanne Maia Pantoja ${ }^{\mathrm{i}}$ \\ Roberta Silva Rizzo ii \\ Bruno Silva Carvalho iii \\ Victor Conde Ferreira ${ }^{\text {iv }}$ \\ Kandarpa Silva Galas ${ }^{\mathrm{v}}$ \\ Francisco Rafael Marciano Fonseca ${ }^{\mathrm{vi}}$ \\ Germana Costa Paixão vii
}

\begin{abstract}
Resumo: Estudos da qualidade do ar de diferentes ambientes fechados vêm merecendo atenção devido principalmente à grande quantidade de substratos favoráveis à ação de agentes biodegradadores, como os fungos anemófilos, tanto sobre os acervos físicos quanto os digitais. A presente pesquisa identificou e monitorou a micobiota aérea de quatro bibliotecas públicas de referência no município de Fortaleza, Ceará, durante 24 meses. A coleta foi realizada pelo método da sedimentação passiva, bimestralmente, em diversos setores das bibliotecas, perfazendo 240 amostras. Após incubação, houve contagem global e identificação das colônias fúngicas baseada nas análises macro e micromorfológicas. Nas bibliotecas A, B, C e D foram contabilizadas 3.347, 5.874, 6.328 e 5.333 colônias, respectivamente, e identificados 61 achados fúngicos (34 gêneros e 27 espécies). Os dados revelaram alguns agrupamentos com forte correlação, a saber: Biblioteca A (Penicillium sp. e Aspergillus sp.), B (A. niger e A. flavus), C (Penicillium sp., Trichoderma sp. e A. niger) e D (Penicillium sp. e A. niger). A pesquisa também permitiu oferecer aos gestores das bibliotecas elementos para aprofundar seus conhecimentos na área de preservação e conservação de obras literárias de inestimável valor.
\end{abstract}

Palavras-chave: Qualidade do ar. Bibliotecas. Micobiota.

\section{CONSTITUTION OF THE AIRBORNE MYCOBIOTA OF PUBLIC LIBRARIES IN THE MUNICIPALITY OF FORTALEZA, CEARÁ, BRAZIL}

\begin{abstract}
It is important to study the air quality of libraries due to the large number of substrates (books, documents, digital media) that are prone to the action of biodegraders, such as airborne fungi. The present study identified and monitored the airborne mycobiota of four public libraries in the municipality of Fortaleza during 24 months. The fungal specimens were collected by passive sedimentation every two months, in various sectors of the libraries, for a total of 240 samples. After incubation, the fungal colonies were counted and identified based on macro- and micro-morphological characteristics. In libraries A, B, C and D there were 3,347, 5,874, 6,328 and 5,333 colonies, respectively, divided into 34 genera and 27 species fungi. The data revealed some clusters with strong correlation, namely: library A (Penicillium sp. and Aspergillus sp.), B (A. niger and A. flavus), $C$ (Penicillium sp., Trichoderma sp. and A. niger) and D (Penicillium sp. and A. niger). The study also provided information to enable library staff to improve the preservation of the valuable literary works and other items in their collections.
\end{abstract}

Keywords: Air Quality. Libraries. Mycobiota.

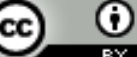

Esta obra está licenciada sob uma Licença Creative Commons

\footnotetext{
${ }^{\mathrm{i}}$ Universidade Federal do Ceará. 1ydiapantoja@bol.com.br.

ii Universidade Estadual do Ceará. robertasilvarizzo@gmail.com.

iii Universidade Estadual do Ceará. brunobsc1@hotmail.com.

${ }^{\text {iv }}$ Universidade Estadual do Ceará. o-conde@hotmail.com.

${ }^{v}$ Universidade Estadual do Ceará. kandarpa.galas@gmail.com.

${ }^{v i}$ Universidade Estadual do Ceará. chicaorafael@yahoo.com.br.

vii Universidade Estadual do Ceará. germanapaixao@terra.com.br.

Recebido em: 14/02/2012; aceito para publicação em: 06/07/2012
} 


\section{INTRODUÇÃO}

Um dos grandes desafios do homem em sociedade é a busca de formas de preservação do patrimônio histórico, artístico e cultural da humanidade. Esse patrimônio é mantido em acervos contidos em locais tecnicamente adequados à sua conservação e de fácil acesso: as bibliotecas (ROSA et al., 2008).

A melhoria desses espaços vem permitindo o avanço da Aerobiologia, a ciência que estuda a produção, a liberação, o transporte e a deposição das partículas biológicas, também chamadas de bioaerossóis. (MAIN, 2003).

Os bioaerossóis são definidos como partículas de origem biológica, suspensas no ar, geradas natural ou artificialmente, que podem existir na forma de célula única, aglomerados de microrganismos viáveis ou partículas não viáveis de vários tamanhos. (PASTUSZKA et al., 2000). São numerosos e diversificados: vírus, bactérias, fungos, cistos de protozoários, grãos de pólen, fragmentos de plantas, insetos, bem como qualquer partícula deles originada.

Estudos sobre a qualidade do ar são importantes fontes de pesquisa nas áreas da saúde pública e ocupacional, principalmente após a descoberta de que baixas taxas de circulação de ar em ambientes fechados, como as bibliotecas, ocasionam um aumento considerável na concentração de poluentes químicos e biológicos. A deterioração de acervos pode ocorrer sob a influência de umidade, temperatura e iluminação inadequadas que favoreçam a presença de fungos e bactérias, a ação de roedores e insetos e a poluição ambiental. (BRICKUS; AQUINO NETO, 1999).

Em especial, o efeito da temperatura e da umidade no ambiente deve ser relacionado. Apesar de atualmente encontrar-se disponível tecnologia sofisticada para controle de tais variáveis, o setor de preservação e conservação de acervos necessita de meios para correlacioná-las diretamente com a qualidade do ar das bibliotecas, particularmente quando o ambiente sofre variações diárias ou mudança de estação climática. (VALLE, 1991; REILLY; NISHIMURA; ZINN, 2001). Nesse ínterim, a literatura reporta que o excesso de umidade apressa a acidificação do papel, enquanto a baixa umidade conduz ao dessecamento, deixando-o quebradiço e frágil. Concomitantemente, essa variação de umidade favorece o desenvolvimento de micro-organismos, com destaque para os fungos. (VALLE, 1991).

Nesse sentido, a colonização do ar depende de condições do ambiente, de forma que os bioaeróssois variam em qualidade e quantidade, podendo ser distintos dois tipos de microbiota aérea: de ambientes fechados e de ambientes abertos. (PASTUSZKA et al., 2000; 
HUANG et al., 2002). Segundo Li e Kuo (1992), os bioaerossóis de ambientes fechados são apontados como uma das principais causas de problemas respiratórios que culminam com a ausência de estudantes da escola e profissionais do trabalho, ou a baixa produtividade em hospitais, bibliotecas e ambientes ocupacionais.

A orientação técnica sobre Padrões Referenciais de Qualidade do Ar Interior em ambientes climatizados artificialmente, de uso público e coletivo, recomenda o monitoramento e controle ambiental de fungos como marcador epidemiológico da contaminação microbiana. (BRASIL, 2000).

Os fungos que comumente habitam o ambiente aéreo são denominados fungos anemófilos ou alergizantes, destacando-se os gêneros Alternaria sp., Aspergillus sp., Cladosporium sp., Curvularia sp., Fusarium sp. e Penicillium sp. (ALEXOPOULOS et al., 1996; HOOG; GUARRO; GENÉ, 2000). Entretanto, dependendo do grau de exposição, outras espécies também podem colonizar o ar.

A literatura especializada descreve que algumas espécies de fungos, frequentemente encontradas no ar em bibliotecas, tais como Aspergillus niger e Aspergillus fumigatus, são corriqueiramente associadas à alergias e micoses respiratórias. (GALLO; GALLO, 1988; MOBIN, 1997; RÊGO; MAGALHÃES; SILVEIRA, 2004).

Estudos liderados por PEI-CHI e colaboradores em 2000 mostraram que a exposição a fungos do ar parece estar associada ao desenvolvimento de várias doenças respiratórias, em especial às asmas ditas "de clima", às quais estão na dependência ou em relação íntima com a microbiota micótica do ar. Ademais, fungos anemófilos também podem estar ligados à gênese de aspergilose, pneumonite por hipersensibilidade, rinite e algumas reações tóxicas. (REPONEN et al., 1996; LACAZ et al., 2002). Existem ainda relatos de funcionários de bibliotecas que desenvolveram rinite alérgica e asma brônquica ao manusearem antigos livros e periódicos (AAS, 1972).

Diante disso, a presente pesquisa objetivou conhecer o espectro fúngico da micobiota aérea de bibliotecas públicas locais e gerar aos seus gestores, elementos para aprofundar seus conhecimentos na área de preservação e conservação, bem como indicar estratégias de prevenção aos danos às obras literárias de inestimável valor. 


\section{MATERIAL E MÉTODOS}

Foram selecionadas quatro bibliotecas públicas de referência no município de Fortaleza, Ceará, denominadas Biblioteca A, Biblioteca B, Biblioteca C e Biblioteca D.

As bibliotecas foram selecionadas por apresentarem as seguintes peculiaridades: a Biblioteca A, fundada em 1880, é constituída de aproximadamente 120.000 livros de registros, classificadores, documentos históricos e revistas, distribuídos em $1.800 \mathrm{~m}^{2}$. A Biblioteca B ocupa área de $200 \mathrm{~m}^{2}$, em um prédio composto por dois andares, onde se encontra distribuído o acervo de aproximadamente 15.000 títulos, voltados para os estudos de línguas e filosofia. A Biblioteca C foi fundada em 1867, ocupa uma área geográfica de $2.272 \mathrm{~m}^{2}$, distribuídos em cinco pavimentos, e apresenta um acervo de aproximadamente 100.000 volumes, incluindo obras raras nacionais. A Biblioteca D, criada em 1975, apresenta uma área de $1.958 \mathrm{~m}^{2}$ e 62.000 exemplares e atende prioritariamente o público de universitários e pós-universitários.

Durante 24 meses (janeiro/2009 a dezembro/2011), bimestralmente, foram analisados cinco setores de cada biblioteca: acervo de obras, área de pesquisa, sala de leitura, sala de documentos históricos e áreas administrativas, como a secretaria, perfazendo 240 amostras biológicas no total.

Para a coleta das amostras provenientes do ar, foi utilizado o método da sedimentação passiva em placas de Petri de $150 \mathrm{~mm}$ de diâmetro, contendo o meio de cultura Agar Batata Dextrose (Himedia $\left.{ }^{\circledR}\right)$. Cada placa foi exposta por 12 horas, das $8 \mathrm{~h}$ às $20 \mathrm{~h}$, sendo colocada a uma altura de 2 metros acima do solo - próximo da área de respiração humana. (PEI-CHIN; HUEY-JEN; CHIA-YIN, 2000).

Findo o período de coleta, as placas de Petri contendo as amostras biológicas foram vedadas e encaminhadas ao Laboratório de Microbiologia (LAMIC) do Curso de Ciências Biológicas da Universidade Estadual do Ceará.

As placas permaneceram incubadas à temperatura de $26-28^{\circ} \mathrm{C}$ durante sete dias, realizando-se observações diárias. A partir do aparecimento de colônias fúngicas procedeu-se a contagem global das mesmas, categorizando-se por setor analisado. Por fim, a identificação das colônias fúngicas ocorreu com base nas análises macro e micromorfológicas. (HOOG; GUARRO; GENÉ, 2000; KONEMAN et al., 2001; SIDRIM; ROCHA, 2004). 
Os dados catalogados permitiram a construção de dendogramas. Os itens com maior correlação foram agrupados considerando a distância de conexão completa. (VALENTIN, 2000).

\section{RESULTADOS E DISCUSSÃO}

A contagem global de colônias de todos os ambientes das bibliotecas A, B, C e D resultou em 3.347, 5.874, 6.328 e 5.333 colônias fúngicas, respectivamente. O número de colônias de cada uma das bibliotecas foi analisado comparativamente e não mostrou relevância estatística $(\mathrm{p} \geq 0,05)$ entre os diferentes ambientes analisados, os tipos de acervos e as áreas físicas.

Essa alta concentração de colônias era esperada, devido principalmente à grande quantidade de substratos favoráveis à ação de biodegradadores/biopoluentes sobre os acervos físicos e digitais. Os dados corroboram com o estudo conduzido por Bortoletto e colaboradores em 2002, em que foi constatada uma séria contaminação fúngica no ar da biblioteca de Manguinhos da Fundação Oswaldo Cruz, cujo acervo contava com 620.000 volumes, na época. A biblioteca foi interditada por 5 meses devido a essa contaminação.

Identificaram-se 61 diferentes grupos fúngicos, distribuídos em 34 gêneros e 27 espécies, com destaque para os gêneros de hialohifomicetos Aspergillus sp. (MICHELI, 1729) e Penicillium sp. (MICHELI, 1729), encontrados nas quatro bibliotecas. Os demais gêneros foram observados com menor frequência, conforme demonstrado na Figura 1. Os hifomicetos não identificados até gênero foram incluídos na ordem Agonomycetales (Mycelia sterilia). A composição do espectro de fungos anemófilos foi formada predominantemente por deuteromicetos filamentosos hialinos.

Visando a análise mais apurada sobre a diversidade dos achados micológicos, foram construídos dendogramas cujos itens com maior correlação (normalmente positiva e direta) foram agrupados, considerando a distância de conexão completa (a maior distância entre os termos), já que foi constatada forte similaridade entre os elementos de cada uma das bibliotecas (Figura 1).

Na Biblioteca A Penicillium sp., Aspergillus niger, Aspergillus sp. e Aspergillus flavus formaram o primeiro agrupamento, e assim sucessivamente. Logo, quando um desses fungos ocorre, os outros três também podem estar presentes no ar da Biblioteca A. Na Biblioteca B 
percebeu-se forte correlação entre o Aspergillus niger e A. flavus. Na Biblioteca C, Penicillium sp., Trichoderma sp. e A. niger, para a Biblioteca D Penicillium sp. e A. niger, todos representando o primeiro agrupamento.

A Biblioteca B apresentou o maior número de agrupamentos (12), seguido das Bibliotecas A e C, com 11 agrupamentos cada, e da Biblioteca D, com o menor número de agrupamentos (8). De acordo com Vicini (2005) o objetivo da construção de dendogramas é agrupar variáveis graficamente, sendo útil para classificar, comparar e discutir agrupamentos. Nesse sentido, foi possível verificar quais achados fúngicos estão mais próximos um dos outros. Com esses dados, os gestores das bibliotecas podem fazer uso de técnicas de preservação específicas, como o uso de fungicidas, quando necessário, ou técnicas gerais, como remover a poeira e eliminar os elementos poluentes, realizar manutenção periódica do aparelho de ar condicionado, permitindo assim manter a integridade dos acervos e garantir que estes tenham uma vida longa. (REILLY; NISHIMURA; ZINN, 2001; CAMPOS, 2006).

Ainda segundo pesquisadores na área de conservação, pode-se afirmar que o crescimento dos fungos comumente encontrados em museus e arquivos acontece com maior intensidade quando a umidade relativa do ar é superior a $65 \%$, bem como as boas práticas de higiene e manutenção do ambiente e do acervo da biblioteca são imprescindíveis para a boa qualidade do ar e para evitar deteriorização das obras. (NIEVES et al., 1998; GUTHS et al., 2002).

A representativa variedade de fungos encontrada coaduna-se a dois estudos anteriores realizados no estado do Ceará. Menezes e colaboradores (2006) expuseram 50 placas de Petri na sala de periódicos da Biblioteca das Ciências da Saúde da Universidade Federal do Ceará e isolaram 13 gêneros fúngicos, com destaque para Aspergillus sp., Penicillium sp., Curvularia sp. e Cladosporium sp., concluindo que aquele espaço era insalubre, já que os fungos poderiam desencadear alergias respiratórias nos seus frequentadores.

A segunda pesquisa sobre o tema conhecida no Ceará foi realizada por pesquisadores do Laboratório de Microbiologia do Curso de Ciências Biológicas da UECE, quando monitoraram o ar da Biblioteca Central do Campus do Itaperi, por um período de um ano e identificaram vários fungos anemófilos, com maior taxa de prevalência para Acremonium blochii, Aspergillus flavus, Aspergillus niger, Aspergillus sp., Fusarium clamidosporium, Fusarium sp., Mycelia sterilia, Mucor sp., Penicillium sp., Rhizopus sp. e Scytalidium hyalinum. (PANTOJA; COUTO; PAIXÃO, 2007). 
Gambale e colaboradores (1993) investigaram a microbiota fúngica do ar e de livros em 28 bibliotecas da Universidade de São Paulo e constataram como fungos mais frequentes no ar, os gêneros Cladosporium, Fusarium, Aspergillus, Rhodotorula, Epicoccum, Aureobasidium, Neurospora, Trichoderma, Rhizopus, Phoma, Monascus, Curvularia, Alternaria, Penicillium, Geotrichum, Acremonium e outros não-esporulados (Mycelia sterilia).

A diversidade do espectro fúngico do ar de bibliotecas situadas em diferentes locais é reforçada ainda com os dados de Rosa e colaboradores (2008), cujo estudo apontou os fungos mais frequentes na biblioteca da Faculdade de Medicina da Universidade Federal de Goiás, que foram os zigomicetos Mucor sp., Rhizopus sp. e Syncephalastrum sp., o que reforça que a distribuição fúngica obedece a um padrão geográfico, enfatizando a importância de estudos regionalizados que visem conhecer a micobiota específica de cada região. 


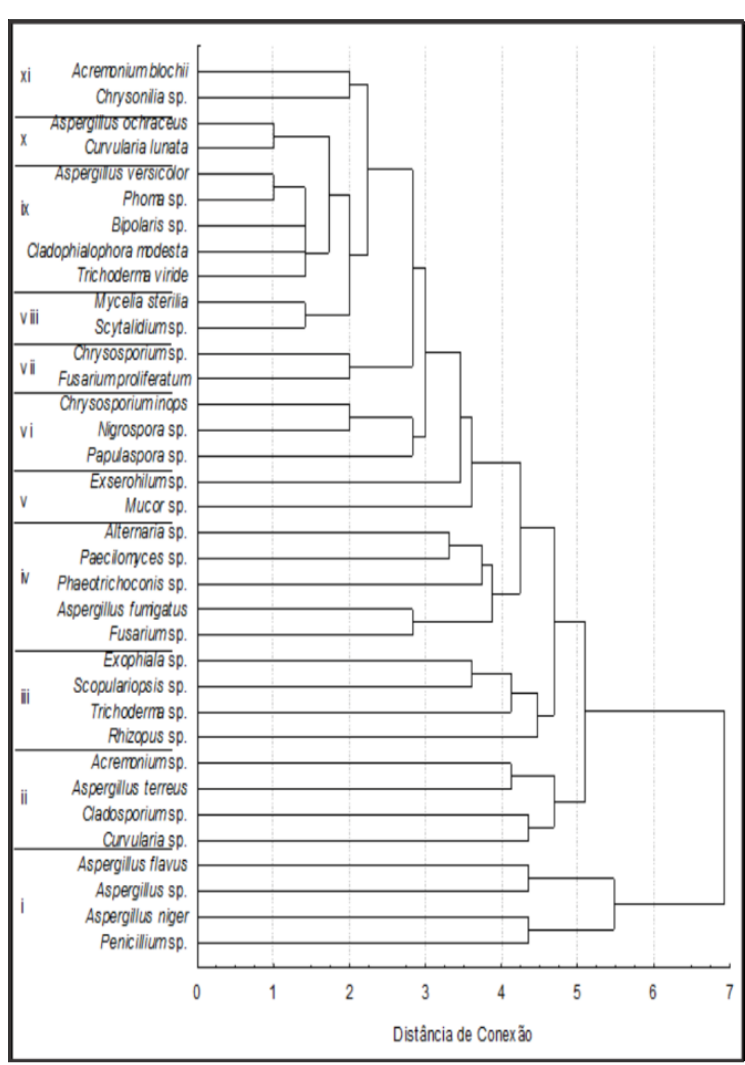

Biblioteca A

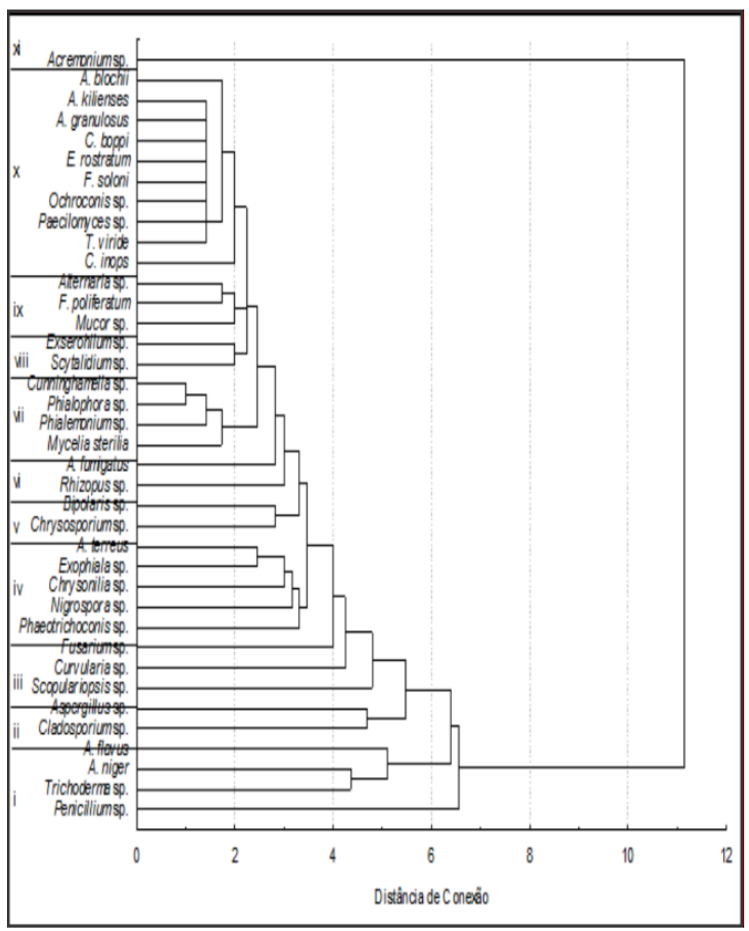

Biblioteca C

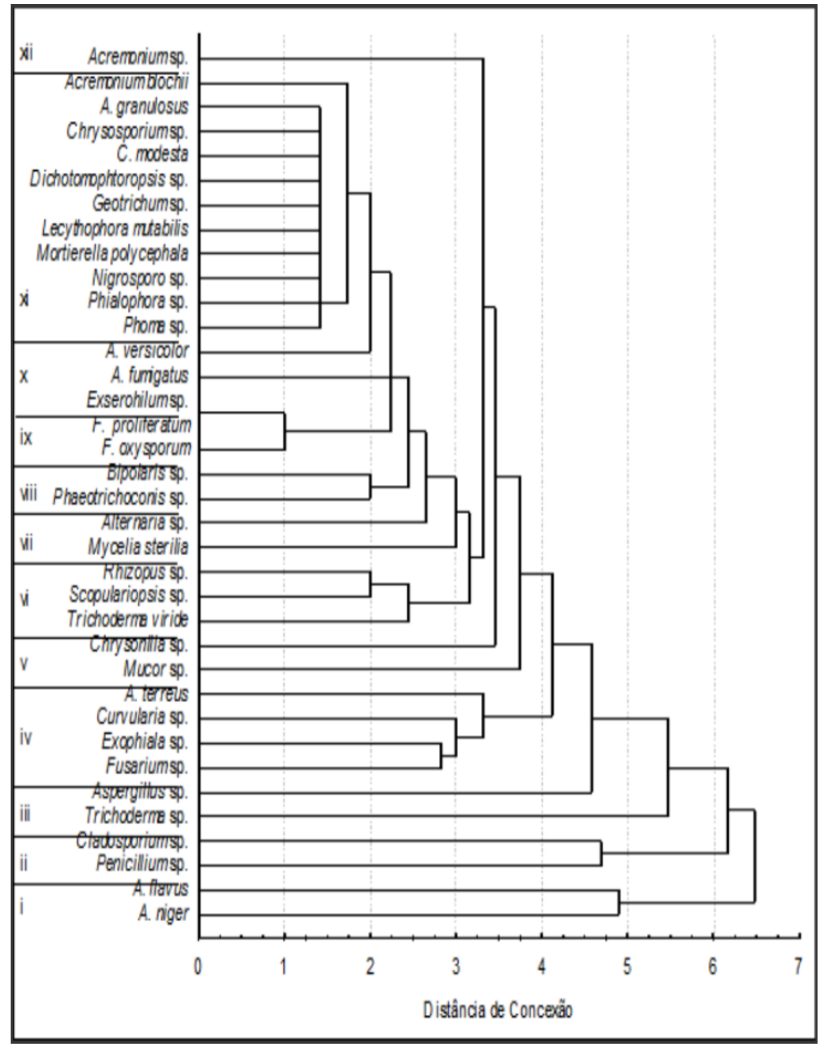

\section{Biblioteca B}

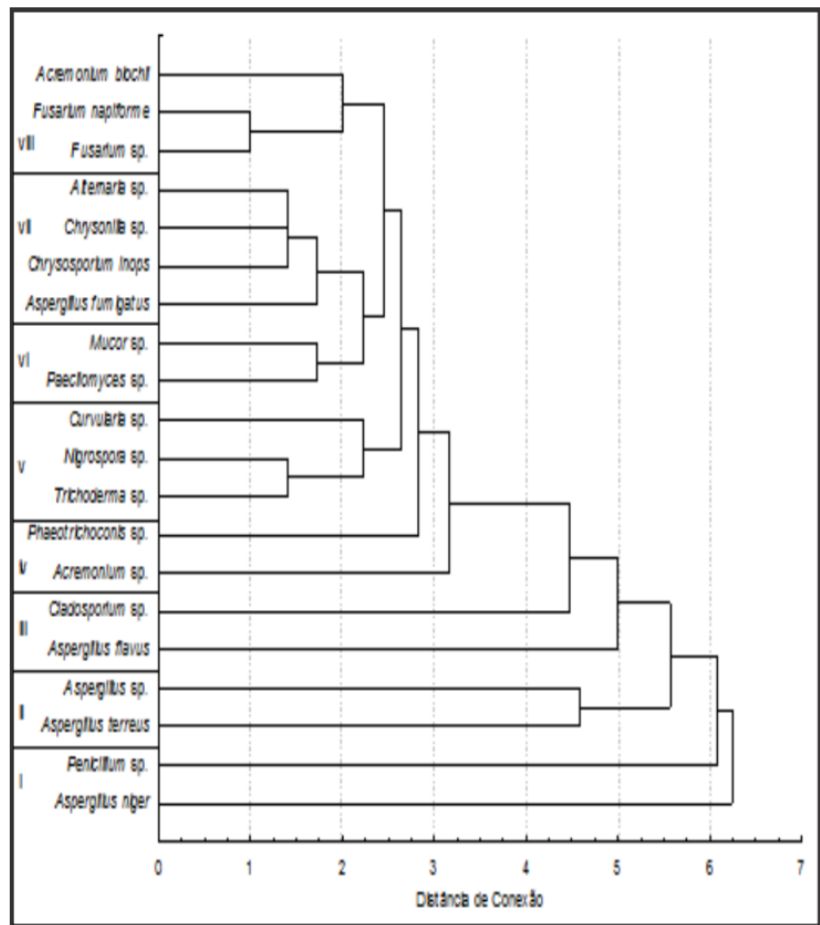

\section{Biblioteca D}

Figura 1. Dendogramas da qualidade do ar das Bibliotecas A, B, C e D. No eixo y, a diversidade de gêneros e espécies fúngicas e, no eixo $\mathrm{x}$, a distância de conexão entre os agrupamentos. 


\section{CONSIDERAÇÕES FINAIS}

Destacamos a significativa diversidade do espectro fúngico encontrado na micobiota aérea das bibliotecas, bem como a existência de agrupamentos com forte correlação, em especial com os hialomicetos Aspergillus e Penicillium. Com base nesses achados pode-se propor que o espaço físico das bibliotecas analisadas passe a ser suficientemente arejado, racionalmente iluminado, limpo periodicamente e que os valores termo-higrométricos sejam adequados à preservação dos livros e documentos.

A despeito da imensurável importância das bibliotecas públicas para o patrimônio cultural da humanidade, nem sempre tais instituições dispõem de corpo técnico habilitado para sua correta manutenção, nem dotações orçamentárias compatíveis, sendo necessária a continuidade de estudos correlacionando datações das obras, tipos de climatização do ambiente, entre outros, sendo fato que muito ainda precisa ser feito frente a qualidade do ar de bibliotecas.

Nesse sentido, o conhecimento e monitoração periódica da micobiota aérea podem nortear a adoção de normas mínimas no trato com as unidades documentais e o espaço físico das bibliotecas, além de contribuir com a compilação de dados epidemiológicos desses aeroalérgenos que podem repercutir ainda na conservação da saúde de seus usuários.

\section{AGRADECIMENTOS}

Ao Prof. Dr. Aldeney Andrade Soares Filho pela análise estatística, aos gestores das bibliotecas por aceitarem a presente pesquisa e a Universidade Estadual do Ceará pela disponibilidade de bolsa de iniciação científica.

\section{REFERÊNCIAS}

AAS, K. The allergic child. In: C. Thomas (Ed.), Chest. Springfield, Illinois. 1972.

ALEXOPOULOS, C. J.; MIMS, C. W.; BLAKWEL, L. Introductory mycology. 4. ed. New York: John Wiley e Sons, 1996. 869 p.

BRASIL. MINISTÉRIO DA SAÚDE. Orientação técnica sobre padrões referenciais de qualidade do ar interior em ambientes climatizados artificialmente de uso público e 
coletivo. Resolução $n^{\circ}$ 176, 24 de outubro de 2000. Brasília: Centro de Documentação do Ministério da Saúde, 2000.

BRICKUS LSR, AQUINO NETO FR. A Qualidade do Ar de Interiores e a Química. [S.1.]: Química Nova 22, 1999. p. 1-10

BORTOLETO, M. E.; MACHADO, R.R.; COUTINHO, E. Contaminação fúngica do acervo da biblioteca de Manguinhos da Fundação Oswaldo Cruz. Ações desenvolvidas para sua solução. Encontros Bibli: revista eletrônica de Biblioteconomia e Ciência da Informação, Florianópolis, v. 14, p. 1-10. 2002.

CAMPOS, M. L. F de. Políticas de preservação de documentos em bibliotecas públicas estaduais brasileiras. $76 \mathrm{f}$. Monografia de graduação. Faculdade de Biblioteconomia e Comunicação da Universidade Federal do Rio Grande do Sul, Porto Alegre, 2006.

GALLO, F.; GALLO, P. Esperienze nel campo della disinfezione e disinfestazione con ossido di etilene. IN: Seminario di studi su tutela dei documenti di archivio, disinfezione e disinfestazione con ossido di etilene, Roma, [s.n.], 1988.

GAMBALE, W.; CROCE, J.; MANSO, E. R. C.; CROCE, M.; SALES, J. M. Library fungi at University of São Paulo and their relationship with respiratory allergies. Journal of Investigative Allergology and Clinical Immunology, v. 3, p. 45-50, 1993.

GÜTHS, S.; KUSTER, S.; LORENZETTII, M. C.; SOUZA, L. A. C. Monitoring system and air supply system to preservation of collections. In: Congresso ABRACOR, 11., 2002, Rio de Janeiro. Anais... Rio de Janeiro, [s.n.], 2002.

HOOG, G. S. de; GUARRO, J.; GENÉ, J.; FIGUEIRAS, M. J. Atlas of Clinical Fungi. 2. ed. Baarn/Delft: Centraalbureau voor Schinmelculture/Universitat Rovira i Virgilli, 2000. 2108 p.

HUANG, C., LEE, C. L. F., MA, Y., SU, H. J. The seasonal distribution of bioaerosols in municipal landfill sites: a 3-yr study. Atmospheric Environment, v. 36, p. 4385-4395. 2002.

KONEMAN, E. W.; ALLEN, S. D.; JANDA, W. M.; SCHRECKENBERGER, P. C.; WINN Jr. W. C. Diagnóstico microbiológico: texto e atlas colorido. 5. ed. Rio de Janeiro: MEDSI, 2001. 2067 p.

LACAZ, C. S.; PORTO, E.; MARTINS, J. E. C.; HEINS-VACCARI, E. M.; MELO, N. T. de. Fungos e Alergia. In: Tratado de Micologia Médica Lacaz. São Paulo: Sarvier, 2002. p. $810-828$.

LI, C. e KUO, Y. Airbone characterization of fungi indoors and outdoors. J. Aerosol Sci, v. 23, p. 667-670, 1992.

MAIN, C. E. Aerobiological, ecological and health linkages. Environment International, v. 29 , n. 2/3, p. 347-349, 2003.

MENEZES, E. A.; ALCANFOR, A. C.; CUNHA, F, A. Airborne fungi in the periodics room of the library of health science of the University Federal of Ceará. Revista Brasileira de Análises Clínicas, v. 38, p. 155-158, 2006. 
MOBIN, M. Myxomycetes e fungos micófilos ocorrentes em palmeiras no Parque Nacional de Sete Cidades (Piripiri - Piauí - Brasil). 116 f. Dissertação de Mestrado. Universidade Federal de Pernambuco, Recife, 1997.

NIEVES, V.; GARCÍA, G.; DE LUIS, O.; MAEKAWA, S. Microbial control in Archives, Libraries and Museums by Ventilation Systems. Restaurator, p. 85-107, 1998.

PANTOJA L. D. M.; COUTO, M. S.; PAIXÃO, G. C. Diversidade de Bioaerossóis presentes em ambientes urbanizados e preservados de um Campus universitário. Biológico, v. 69, p. 4147, 2007.

PASTUSZKA, J. S., PAW, U. K. T., LIS, D. O., WLAZLO, A. ULFIG, K. Bacterial and fungal aerosol in indoor environment in Upper Silesia, Poland. Atmospheric Environment, v. 34, p. 3833-3842. 2000.

PEI-CHIN, W., HUEY-JEN, S., CHIA-YIN, L. Characteristics of indoor and outdoor airbone fungi at suburban and urban homes in two seasons. The Science of the Total Environment, v. 253, p. 111-118. 2000.

RÊGO, R. S. M.; MAGAlHÂES, K.; MELO, F.; SILVEIRA, N. S. S. Ocorrência de Aspergillus sp. em pacientes com sinusite crônica. In: Congresso Brasileiro de Micologia, 4., 2004, Universidade Federal de Ouro Preto, Ouro Preto. Anais... Ouro Preto, [s.n.], 2004. p.135,

REILLY, J. M.; NISHIMURA, D. W.; ZINN, E. Novas ferramentas para a preservação: avaliando os efeitos ambientais a longo prazo sobre coleções de bibliotecas e arquivos. 2. ed. Rio de Janeiro: Projeto Conservação Preventiva em Bibliotecas e Arquivos, 2001.

REPONEN, T.; WILLEKE, K.; ULEVICIUS, V.; REPONEN, A.; GRINSHPUN, S. A. Effect of relative humidity on the aerodynamic diameter and respiratory deposition of fungal spores. Atmospheric Environment, v. 30, p. 3967-3974. 1996.

ROSA, H. LEMOS, J. A.; COSTA,C. R.; SILVA, M. R. R.; FERNANDES, O. F. L. Ocorrência de fungos filamentosos em acervo da faculdade de Medicina da Universidade Federal de Goiás. Revista de Patologia Tropical, v. 37, p. 65-69. 2008.

SIDRIM, J. J. C; ROCHA, M. F. G. Micologia Médica à Luz de Autores Contemporâneos. Rio de Janeiro: Guanabara Koogan, 2004.

VALENTIN, J. L. Ecologia numérica: uma introdução à análise multivariada de dados ecológicos. Rio de Janeiro: Interciência, 2000. 117 p.

VALLE, C. A. Subsídios para uma política de preservação e conservação de acervos em bibliotecas universitárias brasileiras. $118 \mathrm{f}$. Dissertação de mestrado. Universidade de Brasília, 1991.

VICINI, L. Análise multivariada da teoria á prática. 215 f. Monografia de graduação. Universidade Federal de Santa Maria, Santa Maria, 2005. 